\title{
Effect of Quadratic Zeeman Energy on the Vortex of Spinor Bose-Einstein Condensates
}

\author{
Tomoya Isoshima and Sungkit Yip \\ Institute of Physics, Academia Sinica, Nankang, Taipei 11529, Taiwan
}

\begin{abstract}
The spinor Bose-Einstein condensate of atomic gases has been experimentally realized by a number of groups. Further, theoretical proposals of the possible vortex states have been sugessted. This paper studies the effects of the quadratic Zeeman energy on the vortex states. This energy was ignored in previous theoretical studies, although it exists in experimental systems. We present phase diagrams of various vortex states taking into account the quadratic Zeeman energy. The vortex states are calculated by the Gross-Pitaevskii equations. Several new kinds of vortex states are found. It is also found that the quadratic Zeeman energy affects the direction of total magnetization and causes a significant change in the phase diagrams.
\end{abstract}

KEYWORDS: Vortex, Spinor, Bose-Einstein condensate

\section{Introduction}

Quantized vortices in the Bose-Einstein condensate (BEC) of ultracold atoms have been a topic of active research. Since the first realization of the vortex, ${ }^{1}$ a variety of studies have been carried out such as those on the formation of the vortex lattice, bending and oscillation of vortex lines, ${ }^{2}$ vortex rings, and so on. For a review of the early studies, see ref. 3. The interest in vortices in ultracold atoms is also increasing with regard to more complicated systems, such as the BEC interacting with an optical lattice, BEC with dipolar interactions, ${ }^{4-6}$ and molecules and Cooper pairs of Fermions. ${ }^{7}$

Despite these extensive studies on vortices, studies on simpler systems such as the spinor BEC of atoms in a uniform magnetic field ${ }^{8-10}$ remains unachieved. The existence of various vortices has been predicted in the seminal papers by $\mathrm{Ho}^{11}$ and Ohmi and Machida ${ }^{12}$ and was subsequently continued by many works. ${ }^{8-10,13-15}$

In the spinor BEC, a vortex state is inseparably related to the spin texture. By utilizing this relation, it is possible to imprint vorticity without rotating the cloud as proved in the experiments. ${ }^{16}$ By applying a quadrupole (nonuniform) magnetic field, the spin texture of the BEC is forced, resulting in the nucleation of the vortex.

Conversely, for a rotated spinor BEC in a uniform magnetic field or without a magnetic field, many theoretical predictions on the possible spin textures have been made. ${ }^{8,9,13-15}$ In this case, the rotation of the trapping potential causes vorticity that produces a spin texture. In a spinor BEC in a uniform field, the boundary condition and magnetic field gradient do not explicitly control the vorticity. Instead, the internal energy of the vortices determines the vortex configuration. However, the experimental observation of vortices still remains to be performed.

In an optically confined BEC, a dominant effect the linear Zeeman effect- due to the intensity of the magnetic field is cancelled by the conservation of the total magnetization (see ref. 17). Nevertheless, the mag- netic field intensity affects the internal spin through the quadratic Zeeman effect, which was ignored in previous studies ${ }^{8,9}$ on the vortices in the spinor BEC. The quadratic Zeeman energy is proportional to the square of the magnetic field intensity and the local spin.

In this paper, we take into account the quadratic Zeeman energy and present new phase diagrams of vortices calculated by the extended Gross-Pitaevskii equation. It is important to consider the quadratic Zeeman energy because its existence in reality is inevitable. In addition, the quadratic Zeeman energy leads to vortex reconfiguration within fixed values of magnetization $M$ and angular momentum $L$.

Atomic interactions are classified into two types: ferromagnetic and antiferromagnetic (polar). ${ }^{11,12}$ In this study, we treat the condensate of sodium atoms $(F=1)$, which is antiferromagnetic. In the absence of a magnetic field for antiferromagnetic systems, vortices (and their lattices) in which the up and down components are populated are stable. ${ }^{13,15}$ They have been labelled as the vortex of region $\mathrm{II}^{8}$ or the $(1, \times, 0)$ type. ${ }^{9,10}$ In a magnetic field, or in the presence of linear Zeeman energy, various other vortex states are found to be possible. The present work aims to study the effects of the quadratic Zeeman energy on such vortex states and to extend the phase diagrams.

\section{Model}

We simulate the vortex states of the BEC of atomic gases by numerically solving the extended GrossPitaevskii equation. The system is rotated with an angular velocity $\Omega$, and a uniform magnetic field is applied. The calculation is similar to that in ref. 8 , but we take into account the quadratic Zeeman energy. For simplicity, we treat a harmonically trapped two-dimensional system.

We assume the direction of the applied magnetic field and the direction of quantization axis to be the $z$-axis. The vector of the rotation $\Omega$ is also in the $+z$ direction.

The order parameter of the condensate consists of macroscopic wavefunctions $\Psi_{j}$, where $j=0, \pm 1$ are hy- 
perfine projections. The order parameters $\Psi_{j}$ are given by the minimization of a free energy

$$
G=E_{1}+E_{\text {int }}+E_{\text {Zee }}-\Omega L-\mu N,
$$

which consists of the single particle energy $E_{1}$, the interaction energy $E_{\text {int }}$, and Zeeman energy $E_{\text {Zee }}$; they are

$$
\begin{aligned}
E_{1} & =\sum_{j} \int d \boldsymbol{r} \Psi_{j}^{*}\left(-\frac{\hbar^{2}}{2 M_{a}} \nabla^{2}\right) \Psi_{j}+V(\mathbf{r}) n_{j} \\
E_{\mathrm{int}} & =\int d \boldsymbol{r}\left(\frac{c_{0}+c_{2}}{2} n^{2}-\frac{c_{2}}{2}\left|2 \Psi_{1} \Psi_{-1}-\Psi_{0}^{2}\right|\right) \\
E_{\mathrm{Zee}} & =\sum_{j} \int d \boldsymbol{r}\left(-p j n_{j}+q j^{2} n_{j}\right)
\end{aligned}
$$

respectively, where the densities $n_{j}=\left|\Psi_{j}\right|^{2}$ and $n=$ $\sum_{j} n_{j}$, particle number $N=\sum_{j} \int n_{j}(\boldsymbol{r}) d \boldsymbol{r}$, angular momentum $L=-\mathrm{i} \hbar \sum_{j} \int \Psi_{j}^{*} \nabla \Psi_{j} \times \boldsymbol{r} d \boldsymbol{r}$, spin index $j \in$ $1,0,-1$, and trapping potential $V(\mathbf{r})=\frac{m\left(2 \pi \nu_{r}\right)^{2}}{2}\left(x^{2}+y^{2}\right)$. The term proportional to $p(q)$ in eq. (4) represents the linear (quadratic) Zeeman energy.

The minimization of $G$ gives the established GrossPitaevskii (GP) equation for the spinor BEC. ${ }^{11,12} \mathrm{We}$ solve the GP equation numerically for various values of the quadratic Zeeman factor $q$ to investigate its effect on the vortex configurations. The parameters $p$ and $\mu$ are varied to obtain results with various magnetization $M_{z}$ at fixed $N=N_{2}$.

The particle number of the $j$-th component is $N_{j} \equiv$ $\int n_{j}(\boldsymbol{r}) d \boldsymbol{r}$. The normalized angular momentums of the $j$-th component $L_{j} \equiv-\mathrm{i} \hbar \sum_{j} \int \Psi_{j}^{*} \nabla \Psi_{j} \times \boldsymbol{r} d \boldsymbol{r} / N_{j}$ where the spin index $j \in 1,0,-1$ are extensively used to identify vortices. The local and total magnetizations are $m_{\alpha}(\mathbf{r})=$ $\Psi^{\dagger}(\mathbf{r}) F_{\alpha} \Psi(\mathbf{r})$ and $M_{\alpha}=\int m_{\alpha}(\mathbf{r}) d \mathbf{r}$, respectively, where $\alpha=x, y, z$.

A system parameter $\varepsilon$ is defined as follows. From the mass $M_{a}$ of atom, an effective scattering length $a=$ $\left(a_{0}+2 a_{2}\right) / 3$, the interaction parameter $c_{0}=4 \pi \hbar^{2} a / M_{a}$ and harmonic trap frequency $\omega_{0}$, we can calculate a harmonic oscillator length $\lambda_{0}=\sqrt{\hbar / M_{a} \omega_{0}}$. We use a ratio of oscillator length to condensate radius $R_{\mathrm{TF}}$, $\varepsilon=\left(\lambda_{0} / R_{\mathrm{TF}}\right)^{2}$ as a system parameter. Here the radius $R_{\mathrm{TF}}$ is that of condensate within Thomas-Fermi (TF) approximation in the absence of spin interaction. For the system parameter, values $\varepsilon=0.1$ and 0.01 are used. After a $\varepsilon$ is given, we have a particle number $N_{2 \mathrm{D}}=1 /\left(16 \epsilon^{2} a\right)$ within the TF approximation. We fix the particle number $N$ to $N_{2 \mathrm{D}}$. The approximate peak density becomes $n_{\text {peak }}=h \nu_{r} /\left(2 c_{0} \varepsilon\right)$ where $h$ is Plank's constant. For sodium atom when $\nu_{r}=100 \mathrm{~Hz}$, $n=0.329(3.29) \times 10^{14} \mathrm{~cm}^{-3}$ for $\varepsilon=0.1(0.01)$.

Value $\varepsilon=0.1$ is used for consistency with our previous study. ${ }^{8}$ Another value $\varepsilon=0.01$ is employed to simulate thin disk-shaped condensate. If we assume tight harmonic confinement and Gaussian shape of condensate wavefunction along $z$-axis, the particle number in a system is $N_{3 \mathrm{D}}=\sqrt{\frac{\hbar \pi}{M_{a} \omega_{z}}} N_{2 \mathrm{D}}$ where $\omega_{z}$ is the confinement parameter along $z$-axis. For sodium atoms when $\omega_{z}=1000 \times 2 \pi$ and $\epsilon=0.01, N_{3 \mathrm{D}}$ is $2.65 \times 10^{5}$. This is within typical experimental particle number.

In an experiment ${ }^{18}$ without a vortex, $q$ ranges from 0 to $70 \mathrm{~Hz}$ for magnetic fields $B_{0}$ up to $500 \mathrm{mG}$. We choose $q /\left(h \nu_{r}\right)$ from 0 to 0.1 . The maximum 0.1 corresponds to $190 \mathrm{mG}$ when $\nu_{r}=100 \mathrm{~Hz}$ and $q \propto B_{0}^{2}$.

The parameters that we explicitly give its values are the system parameter $\varepsilon$, the angular velocity $\Omega$ and the quadratic Zeeman factor $q$. The total magnetization $M_{z}$ and the particle number $N$ are controlled via the linear Zeeman factor $p$ and chemical potential $\mu$. We employ the ratio of the interaction parameters $c_{2} / c_{0}=0.02$, which is obtained from an experiment ${ }^{18}$ that directly treats the quadratic Zeeman energy and interaction parameter. Similar to that experiment, we assume the condensate of sodium atoms with $F=1$.

There are a number of vortex states at each $M_{z}$. Among them, we assume that a state with the lowest energy $G$ is realized. These lowest energy states are presented in the phase diagrams -Figs. 1(a), 1(b) and 4. However, the direction of the total magnetization requires further explanation before the details of the phase diagrams are examined.

\section{Phase diagrams}

\subsection{Direction of total magnetization}

Total magnetization is conserved in an optically confined condensate (see refs. 17, 18). For this conservation, the factor $p$ of the linear Zeeman energy $-p M_{z}$, which was originally proportional to the magnetic field, is treated as a Lagrange multiplier. Therefore, the factor $p$ loses its direct correspondence with the magnetic field. Conversely, the quadratic Zeeman factor $q$, which is proportional to the square of the magnetic field, is not restrained by the conservation.

The horizontal axis in the diagrams is the normalized magnetization $M_{z} / N$ in the $z$-direction. The total magnetization $M_{x}, M_{y}, M_{z}$ may be rotated around $z$-axis by the spin rotation $\exp ^{-i F_{z} \tau}$, without affecting the Zeeman energies. Therefore, elements $M_{x}$ and $M_{y}$ are denoted by $M_{\perp}=\sqrt{M_{x}^{2}+M_{y}^{2}}$ hereinafter.

The amplitudes of the elements $m_{x}$ and $m_{y}$ of the local magnetization, and therefore the amplitude of $M_{\perp}$, are proportional to the amplitude $\left|\Psi_{0}\right|$ of the zero species. Because the zero species of the condensate becomes energetically favorable for larger $q$, the total perpendicular magnetization $M_{\perp}$ can be large for some types of vortices.

Usually, the total magnetization $M_{\perp}$ perpendicular to the $z$-axis is zero. However, in some regions, vortices with $M_{\perp} \neq 0$ have the lowest energy. The regions containing vortices with $M_{\perp} \neq 0$ are also shown in Figs. 1(b) and 4 as the area shaded with (blue) slant lines.

\subsection{Absence of quadratic Zeeman term}

In the absence of the quadratic Zeeman energy $(q=0)$, our system is equivalent to that in ref. 8 at $c_{2} / c_{0}=0.02$. In Fig. 1(a), regions IV, II and III, which have been reported before, ${ }^{8}$ are observed. The boundaries of these 


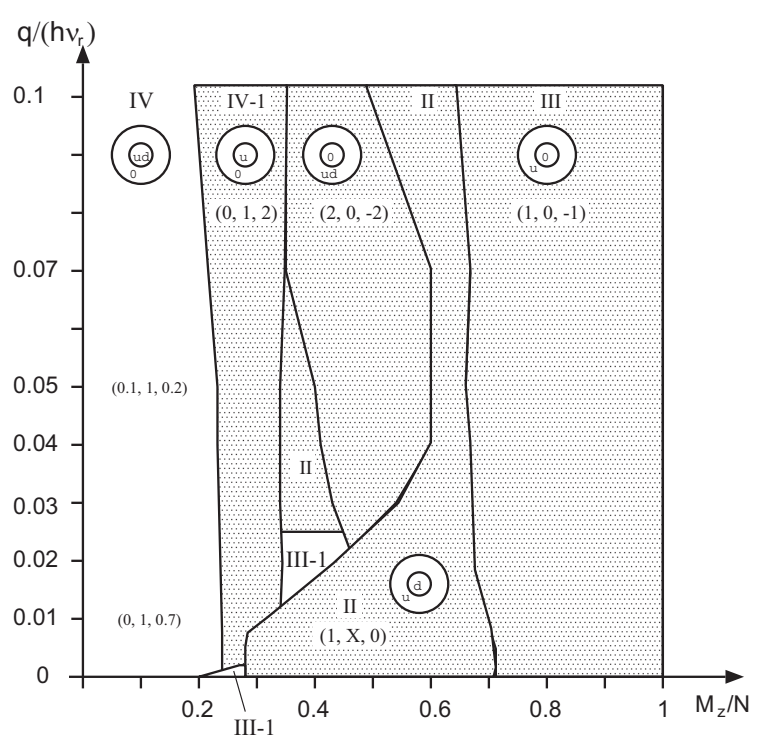

(a)

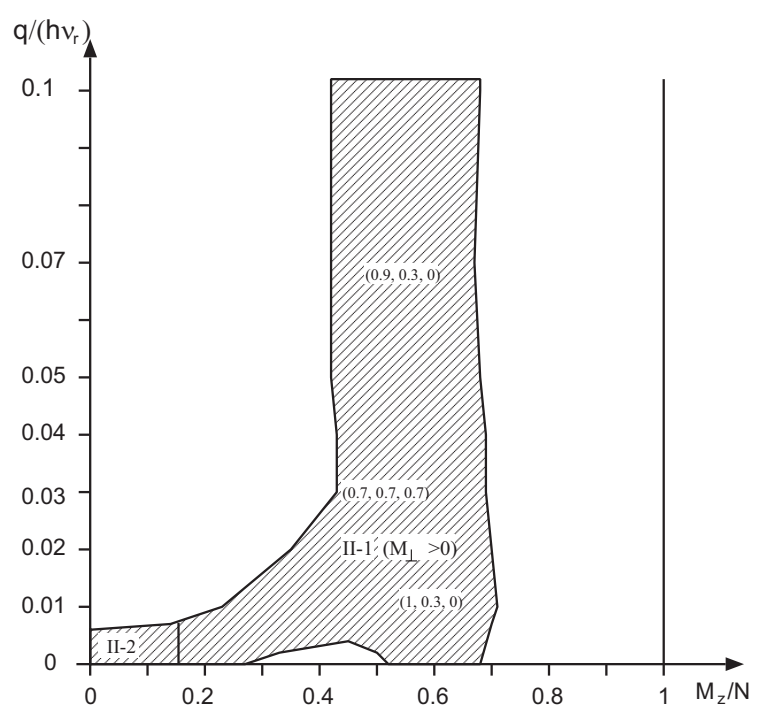

(b)

Fig. 1. Phase diagrams of magnetization $M_{z} / N$ vs the quadratic Zeeman term parameter $q /\left(h \nu_{r}\right)$ at $\epsilon=0.1$ and $\Omega=0.45$. (a) This diagram shows the areas for the vortices with $M_{\perp}=0$. A set of three numbers in parentheses e.g., $(1,0,-1)$ represents the angular moments $L_{1}, L_{0}$ and $L_{-1}$ of the condensate, respectively. In a shaded region, when the numbers in the set are integers, all the vortex states in that region has that set of angular moments. Otherwise, a set of numbers shows only one typical value in the region. Letters $\mathrm{u}, 0$, or $\mathrm{d}$ in double circles indicates that the up, zero, or down species is dominant inside (inner circle) or outside (outer circle) the centered vortex core. (b) Regions II-1 and II-2 in which vortex states with $M_{\perp} \neq 0$ have a lower energy are shown. The perpendicular magnetization $M_{\perp} / N$ in II-1 lies between $0.001 \sim 0.2$ at $q / \nu_{r}>0$. At $q=0$ and $M_{z} / N>0.5$ within II- $1, M_{\perp} / N$ is as low as $10^{-8}$.

regions are consistent with the present results. However, there are also vortex regions II-1 and II-2 exist with $M_{\perp} \neq 0$ and a region III-1 also exists with $M_{\perp}=0$. Figures 1(a) and 1(b) show phase diagrams along the horizontal axis $(q=0)$. Let us consider these regions from smaller $M_{z}$ values at $M_{\perp}=0$. Amplitudes of $\left|\Psi_{i}\right|$

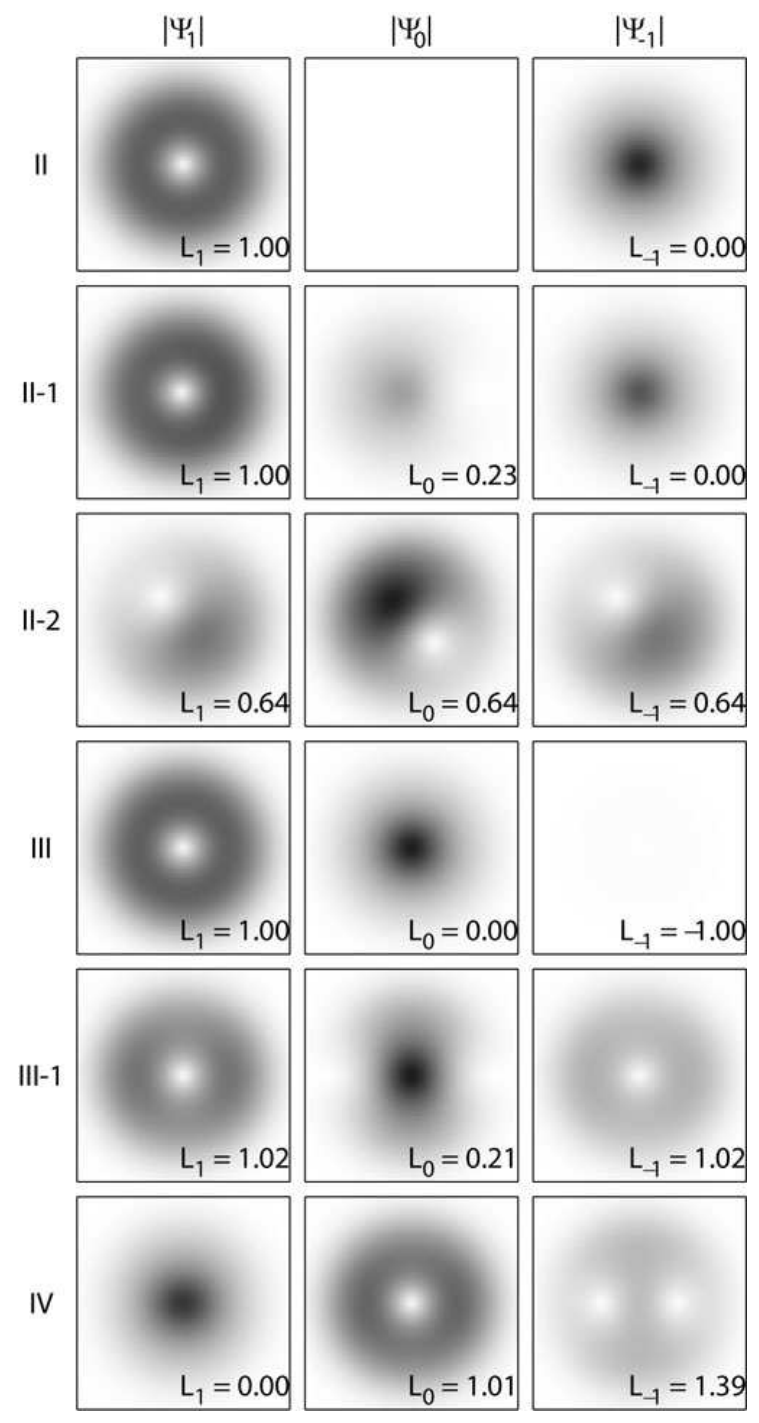

Fig. 2. Amplitudes of $\left|\Psi_{i}\right|(i=0, \pm 1)$ of vortices in regions II, II-1, II-2, III, III- 1 and IV at $\epsilon=0.1$ and $\Omega=0.45 . L_{i}$ are the normalized angular moments for each component.

of the corresponding vortices are shown in Fig. 2.

IV, IV-1: The zero species has a vortex $\left(L_{0} \sim 1\right)$ and the other species fill the core region. Moreover vortex IV-1 has integer angular momentums $\left(L_{1}, L_{0}, L_{-1}\right)=$ $(0,1,2)$. For a simple explanation, let us consider the cases with a single centered vortex on one or two of the three components, and that the other component(s) fillx the vortex core. For a smaller magnetization $M_{z}<0.2$, the local magnetization $m_{z} / n=\left(n_{1}-n_{-1}\right) / n$ tends to be zero. When the local magnetization $m_{z} / n$ is zero, there are two possible configurations: (1) There is a vortex in the zero component, and a mixture of up and down species fills the vortex core, and (2) the mixture has a vortex (up and down species are co-rotating), and the zero species fills the core. Here, under the phase conditions introduced below in eq. (7), (1) is preferred. Hence, type IV is realized. As $M_{z}$ is increased from zero, the angular momentum $L_{-1}$ smoothly increases to fully satisfy eq. (7) in the IV-1 area.

III-1: As mentioned above (2), the up and down species 
exhibit vorticity $L_{1}$ and $L_{-1} \sim 1$, and the zero species fill the core.

II and III: The total angular momentum around $L=$ 0.7 is preferred due to the angular velocity $\Omega$. Therefore, the dominant species should have one vortex. For a larger $M_{z}$ (>0.2), throughout the II and III regions, the up component is dominant and has one vorticity $L_{1}=1$. In region II (III), the down (zero) component fills the core to decrease (increase) $M_{z}$. The type II and III vortices correspond to $(1, \times, 0)$ and $(1,0,-1)$ in ref. 10 , respectively. In this notation, the (integer) angular moments of each component are listed in parentheses for the axisymmetric cases.

Let us consider the vortices with finite $M_{\perp}$ as shown in Fig. 1(b). As compared to the corresponding vortex states in Fig. 1(a), the vortex states in Fig. 1(b) have a lower energy $G$. The II-1 and II-2 regions are shown in Fig. 1(b), and these two types of vortices can be smoothly deformed into each other.

From $M_{z} / N=0$ to 0.276 : For specific value of the parameter $p=0$, a configuration of two vortices is possible. This includes the "split-(I)" states in ref. 10. In our case, the vortex state has $M_{z} / N=0$ and $M_{\perp} / N=$ 0.276 . Because the Zeeman factors are zero, the direction of spin may be freely rotated by the operators $\exp ^{-i F_{x} \alpha} \exp ^{-i F_{y} \beta}$. By this rotation, this vortex is reduced to a type II vortex with $M_{\perp} / N=0, M_{z} / N=$ 0.276 . By similarly continuing the rotation, these vortices cover a range of $M_{z} / N=0-0.276$ while maintaining $\sqrt{M_{\perp}^{2}+M_{z}^{2}} / N=0.276$. Similar deformations of the density profiles among the spin components due to changes in the quantization axis are reported in refs. 8,15 . This deformation affects the energy when the Zeeman term factors $p, q$ are finite, and thus no special care is required in other areas of the phase diagrams.

From $M_{z}=0.51$ to 0.66 : The vortex states in this range have a small finite perpendicular magnetization $M_{\perp} / N \sim 10^{-8}$, and these are similar to the vortices within $0<M_{z}<0.276$. Nevertheless, these cannot be reduced to vortices with $M_{\perp}=0$ because of the finite Zeeman term factor $p$. This region is discussed in $§ 3.4$.

\subsection{Effects of quadratic Zeeman term}

Let us consider the effects of the quadratic Zeeman energy shown in eq. (4). This energy is proportional to the parameter $q$. An increase in the Zeeman energy for a finite $q$ is $q\left(N_{1}+N_{-1}\right)=q\left(N-N_{0}\right)$. Therefore, there is a maximum increase in the energy of vortex of type II, whose $N_{0}=0$; it becomes less favorable. In the phase diagram, the type II region shrinks above $q /\left(h \nu_{r}\right)=0.05$, and a large range of $M_{z}$ is occupied by the other regions. The other boundaries of the regions are not sensitive to $q$.

The non-axisymmetric region III- 1 exists at a finite $q$. In region III-1, angular moments $L_{1}$ and $L_{-1}$ have the same sign and a similar amplitude. In other words, the up and down species are co-rotating in region III-1, while they are counter-rotating in region III. The $c_{2}$ term of the energy prefers the counter-rotating configuration, see

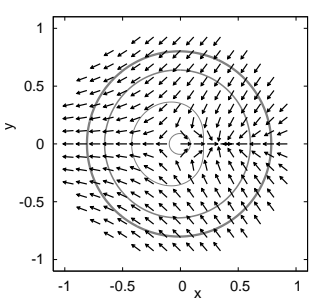

(a)

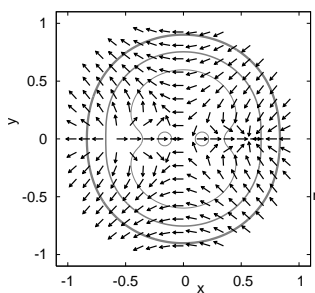

(c)

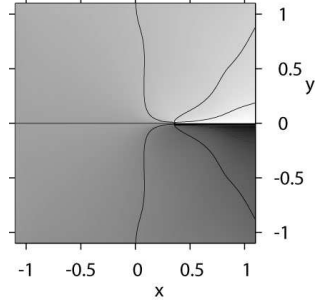

(b)

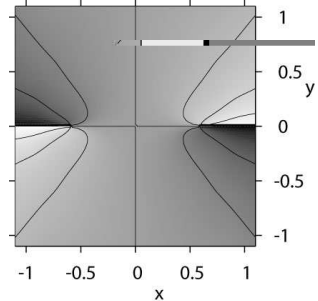

(d)
Fig. 3. (a)(b) A vortex state within the region II-1 at $\varepsilon=0.01$, $q=0.06 h \nu_{r}, M_{z} / N=0.437$ and $M_{\perp} / N=0.080$. (c)(d) A vortex state within the region III-2 at $\varepsilon=0.01, q=0.05 h \nu_{r}, M_{z} / N=$ 0.302 and $M_{\perp} / N=0.085$. (a)(c) Spin textures. Arrows show the directions of local spin components $m_{x}$ and $m_{y}$. Component $m_{z} / n$ is shown by contours. (b)(d) Phases of $\Psi_{0}$. Solid lines indicate multiples of $\pi / 4$.

discussion in $§ 3.4$. However, the co-rotating configuration becomes energetically favorable by decreasing the energy $-\omega L$ because the total angular momentum $L \sim\left(N_{1}+\right.$ $\left.N_{-1}\right) / N$ is larger than $L=\left(N_{1}-N_{-1}\right) / N$ in the counterrotating case.

Several data points show the III-1 configurations even within the region II on Fig. 1(a). Their energies are lower than the type II vortices. But we couldn't make sure that they (III-1) always exist within certain range of $M_{z}$ because they become unstable by small changes in $p$.

\subsection{Phase in the region II-1}

In the region II-1, the phases and angular momentums of the up and down species are similar to those in the region II. However, the wavefunction $\Psi_{0}$ is nonzero there. This minor change in the wavefunction significantly changes the spin texture because the components of local magnetization $m_{x}$ and $m_{y}$ are proportional to the amplitude $\left|\Psi_{0}\right|$. The spin texture in the $x y$ plane appears as shown in Fig. 3(a). The phase of $\Phi_{0}$ is important for understanding of this spin texture.

The phase of $\Psi_{0}$ is restricted by the minimization of the local spin interaction energy of $E_{\text {int }}$ in eq. (3). The $c_{2}$-proportional part of the local energy is written as

$$
\begin{aligned}
\mathcal{E}_{c 2} & =\frac{c_{2}}{2} n+\frac{c_{2}}{2}\left(n^{2}-\left|2 \Psi_{1} \Psi_{-1}-\Psi_{0}^{2}\right|\right) \\
& =C+2 c_{2} \sqrt{n_{1} n_{-1}} n_{0} \cos \left(2 \alpha_{0}-\alpha_{1}-\alpha_{-1}\right)
\end{aligned}
$$

where $\alpha_{j}$ are the phases of $\Psi_{j}$, and $C$ is a function of densities $n_{j}$. In our antiferromagnetic system $\left(c_{2}>0\right)$, a condition for minimizing $\mathcal{E}_{c 2}$ for given $n_{j}$ is

$$
2 \alpha_{0}-\alpha_{1}-\alpha_{-1}=\pi+2 m \pi
$$

where $m$ is an integer. 


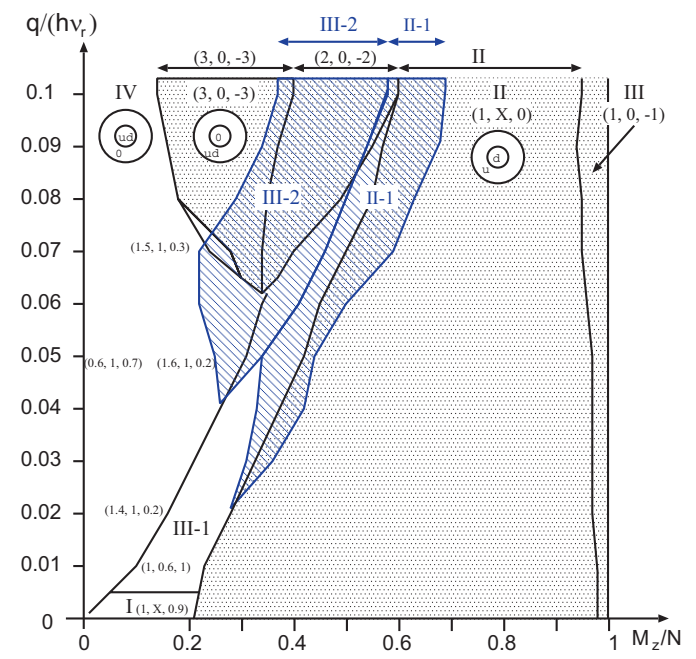

Fig. 4. (Color online) Phase diagram of magnetization $M / N$ vs quadratic Zeeman term $q /\left(h \nu_{r}\right)$ at $\epsilon=0.01$ and $\Omega=0.07$. Notations are same as in Figs. 1(a) and 1(b). But both $M_{\perp}=0$ regions and $M_{\perp} \neq 0$ regions (shaded with slash lines) are shown here. A region at $q=0.07, M_{z} / N \sim 0.25$ is not labelled. There angular momentums are around $(2.7,0.3,0.3)$.

In regions II and II- $1, \alpha_{1} \simeq \theta, \alpha_{-1}=0$. Therefore, $\alpha_{0}=\frac{\theta}{2}$ and the zero species must have a half winding number. In region II-1, instead of a half winding number, the zero species has an off-centered vortex core and a non-integer angular momentum $0<L_{0}<1$, see Fig. 3(b).

The components of the magnetization $m_{x}$ and $m_{y}$ are proportional to the amplitude $\left|\Psi_{0}\right|$. In II-1, because the amplitude $\left|\Psi_{0}\right|$ becomes finite, the spin texture in the $x y$ plane appears as shown in Fig. 3(a).

In the absence of Zeeman terms $p=q=0$, this structure may be reduced to vortices in region IV by changing the quantization axis. At a finite $p$ or $q$, these vortices cannot be reduced similarly and are energetically more favorable than type II vortices. Therefore, the II-1 vortex is likely to be observed in experiments conducted with finite magnetic fields.

\subsection{Disk-shaped condensate}

Figure 4 is the phase diagram as $\varepsilon=0.01$. Amplitudes of $\left|\Psi_{i}\right|$ of the corresponding vortices are shown in Fig. 5. This value corresponds to the realistic particle number for tight $z$-confinement traps. We employ the angular velocity $\Omega=0.07 \times 2 \pi$ to cover the vortex states observed at $\varepsilon=0.1$. At a lower (higher) rotation, $\Omega=0.05 \times 2 \pi$ $(0.1 \times 2 \pi)$, vortexfree (many vortex) states replace these vortex states.

At $q=0$, regions I, II and III are observed. Region I is reported in ref. 8. The composite vortex in this region consists of two vortices, one is in the up component and the other is in the down component. The zero component does not have an atom. The composite vortex in region $\mathrm{I}$ at $p=q=0$ is equivalent to that in region IV through the $\pi / 2$ rotation of the quantization axis. The other regions III and IV are similar to the abovemen-

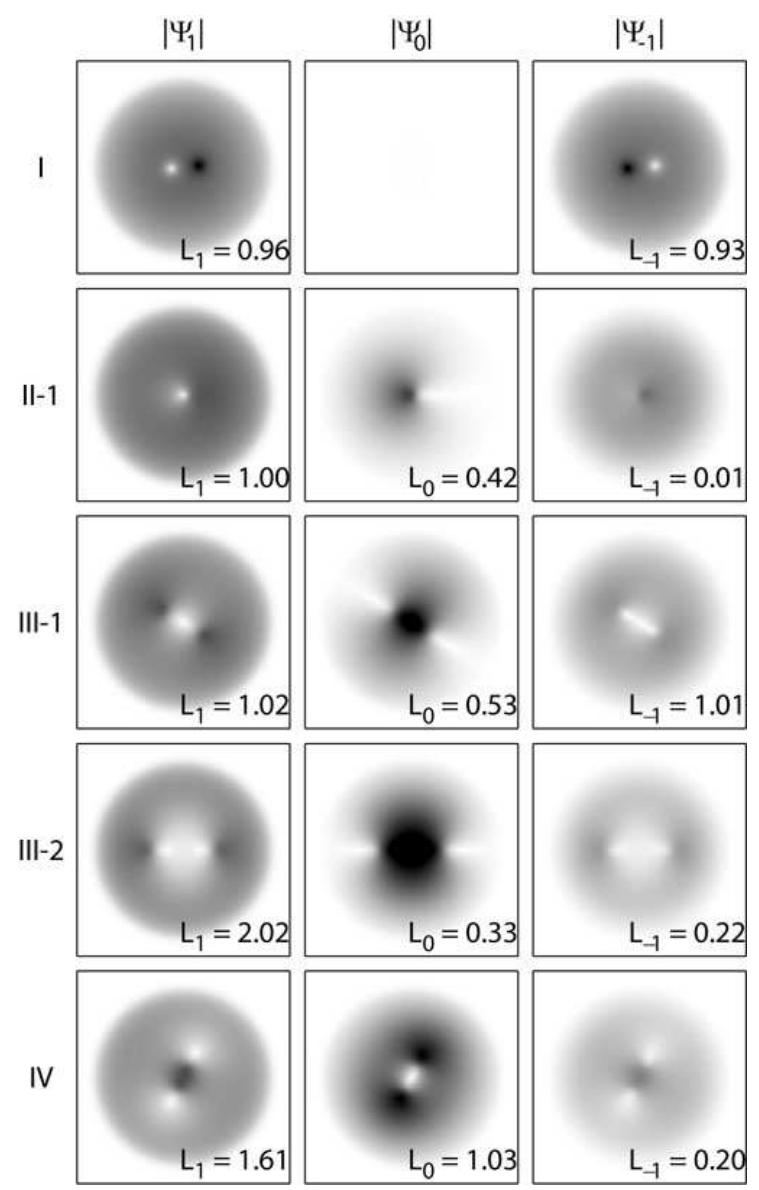

Fig. 5. Amplitudes of $\left|\Psi_{i}\right|(i=0, \pm 1)$ of vortices in regions I, II, III, III- 1 , IV and IV-1 at $\epsilon=0.01$ and $\Omega=0.07$. The normalized angular momentum $L_{i}$ is shown in each panel.

tioned $\varepsilon=0.1$ case.

At finite $q$, because the absence of the zero component is energetically unfavorable, region I is replaced by region III-1. The vortices in region III-1 may be understood as those in region I with finite atoms of zero species.

Regions II, III, III-1 and IV are similar to the $\varepsilon=$ 0.1 case. However, the region II elongates toward larger $q$ because the scale of $\varepsilon$ changes with $q$. Consider two energy densities at the trap center $\mathcal{E}_{c 2} \sim c_{2} n^{2} \propto c_{2} \varepsilon^{-2}$ and $\mathcal{E}_{q} \sim q n \propto q \varepsilon^{-1}$, where $n$ is the atom density. These energies determine the relative population of the zero species, and they should be comparable at the boundary of region II. When we consider the boundary between systems with different $\varepsilon$ values, systems with equal $q \varepsilon$ rather than $q$ are in correspondence.

In regions III, $(2,0,-2)$ and $(3,0,-3)$, the up and down species are counter-rotating with respect to each other (angular moments $L_{1}$ and $L_{-1}$ have opposite sign but the same amplitude). These satisfy the phase relation given by eq. (7). In region III-1, this condition is not met. Region III-1, in which the up and down species are co-rotating, lies in between regions IV and II.

Vortices with finite perpendicular magnetization $M_{\perp}$ reduce the energy at larger $p$. They are shown as areas shaded with oblique lines, e.g., II-1 and III-2 regions. 
The II- 1 region at $M_{z} / N \sim 0.5$ that also appeared when $\epsilon=0.1$. Here, $M_{\perp} / N \sim 0.03-0.09$. Another region is III-2, in which $M_{\perp} / N \sim 0.07$. A pair of spin structures similar to those of the type II-1 vortices exist at $\varepsilon=0.1$.

When the initial perpendicular magnetization is zero $\left(M_{\perp} / N=0\right)$ and the total magnetization is explicitly conserved, only the vortex configurations with $M_{\perp} / N=$ 0 are selected. If there is an error in $M_{\perp} / N$, the II- 1 or III-2 type of vortices having lower energy will be realized. Therefore, determining whether the areas shaded by oblique lines should be considered in this phase diagram depends on the experimental details.

The vortices in region III-2 have a pair of the linelike phase singularities on the zero species as shown in Fig. 3(d). Figure 3(c) presents resulting spin texture. Between the singularities, the center of the trap is filled by zero species. These vortices are regarded as a pair of type II vortices that has gained finite zero species $\Psi_{0}$.

\section{Conclusion}

We investigated the effect of the quadratic Zeeman energy on various vortex states of an antiferromagnetic spinor BEC. We presented phase diagrams showing the quadratic Zeeman term factor $q$ vs magnetization $M_{z} / N$. An important observation is that the perpendicular magnetization $M_{\perp} / N$ is also necessary to correctly plot the phase diagram, not only at finite $q$ but also at $q=0$. The permissible range of $M_{\perp}$ will depend on the experimental conditions.

In a specific case $(p=q=0)$ in which the Zeeman terms are ignored, the rotation of all the spins deforms a vortex in region II into other configurations (regions II-1 and II-2) with a finite perpendicular magnetization $M_{\perp}$. This covers the range $0 \leq M_{z} / N \leq 0.276$ while maintaining $\sqrt{M_{z}^{2}+M_{\perp}^{2}} / N=0.276$.

For a finite $q$, due to the quadratic Zeeman energy, vortices with $M_{\perp} \neq 0$ (in regions II-1, II-2 and III-2) widely replace the other vortices and change the phase diagram. In the general case $(p \neq 0$ or $q \neq 0)$, configurations with finite $M_{\perp}$ cannot be reduced into configurations with $M_{\perp}=0$. Axisymmetric vortices $(3,0,-3)$ and $(2,0,-2)$ whose up and down components are counterrotating with respect to each other are also found at larger $q$.

In the absence of the quadratic Zeeman term $(q=$
0 ), the result is consistent with previous studies by one of us. ${ }^{8}$ However, vortices II-1 and III-1 are also found. These vortices may be understood as a deformation of the vortices in regions II and I with finite zero species.

The vortices in region II are commonly known for the antiferromagnetic spinor BEC. ${ }^{10,13,15}$ For a finite $q$, these vortices gains zero species and are deformed into II-1 vortices. Similarly, III-2 vortices are regarded as derived from a pair of type II vortices. A similar deformation of vortices by having finite zero component is expected in the spinor BEC in a magnetic field, especially in the lattices of the type II vortices. ${ }^{15}$

1) M. R. Matthews, B. P. Anderson, P. C. Haljan, D. S. Hall, C. E. Wieman, and E. A. Cornell, Phys. Rev. Lett. 83 (1999) 2498.

2) V. Bretin, P. Rosenbusch, F. Chevy, G. V. Shlyapnikov, and J. Dalibard, Phys. Rev. Lett. 90 (2003) 100403.

3) A. L. Fetter and A. A. Svidzinsky, J. Phys.: Condens. Matter 13 (2001) R135.

4) A. Griesmaier, J. Werner, S. Hensler, J. Stuhler, and T. Pfau, Phys. Rev. Lett. 94 (2005) 160401.

5) N. R. Cooper, E. H. Rezayi, and S. H. Simon, Phys. Rev. Lett. 95 (2005) 200402.

6) J. Zhang and H. Zhai, Phys. Rev. Lett. 95 (2005) 200403.

7) M. W. Zwierlein, J. R. Abo-Shaeer, A. Schirotzek, C. H. Schunck, and W. Ketterle, Nature 435 (2005) 1047.

8) S.-K. Yip, Phys. Rev. Lett. 83 (1999) 4677.

9) T. Isoshima and K. Machida, Phys. Rev. A 66 (2002) 023602.

10) T. Mizushima, K. Machida, and T. Kita, Phys. Rev. A 66 (2002) 053610.

11) T.-L. Ho, Phys. Rev. Lett. 81 (1998) 742.

12) T. Ohmi and K. Machida, J. Phys. Soc. Jpn. 67 (1998) 1822.

13) J.-P. Martikainen, A. Collin, and K.-A. Suominen, Phys. Rev. A 66 (2002) 053604.

14) T. Mizushima, N. Kobayashi, and K. Machida, Phys. Rev. A 70 (2004) 043613.

15) E. J. Mueller, Phys. Rev. A 69 (2004) 033606.

16) A. E. Leanhardt, Y. Shin, D. Kielpinski, D. E. Pritchard, and W. Ketterle, Phys. Rev. Lett. 90 (2003) 140403.

17) M.-S. Chang, C. D. Hamley, M. D. Barrett, J. A. Sauer, K. M. Fortier, W. Zhang, L. You, and M. S. Chapman, Phys. Rev. Lett. 92 (2004) 140403.

18) J. Stenger, S. Inouye, D. M. Stamper-Kurn, H.-J. Miesner, A. P. Chikkatur, and W. Ketterle, Nature 396 (1998) 345.

19) M. D. Barrett, J. A. Sauer, and M. S. Chapman, Phys. Rev. Lett. 87, 010404 (2001).

20) E. N. Bulgakov and A. F. Sadreev, Phys. Rev. Lett. 90 (2003) 200401. 